\title{
Current transfers
}




\section{Summary}

The deficit for current transfers almost doubled between 1993 and 2000, growing from $f 5.1$ billion in 1993 to $\mathrm{f9.8}$ billion in 2000 .

After decreasing to $\mathrm{f6.5}$ billion in 2001, the deficit increased again in each of the six subsequent years, to $\mathrm{f13.7}$ billion at the end of 2007 - the highest cash figure on record.

The deficit on government transfers widened, from a deficit of $\mathrm{f9.5}$ billion in 2006 to a deficit of $\mathrm{f9.8}$ billion in 2007. Over the same period the deficit for other sectors increased from f2.4 billion in 2006 to $\mathrm{f3.9}$ billion in 2007.

\section{Figure 5.1}

\section{Current transfers}

f billion

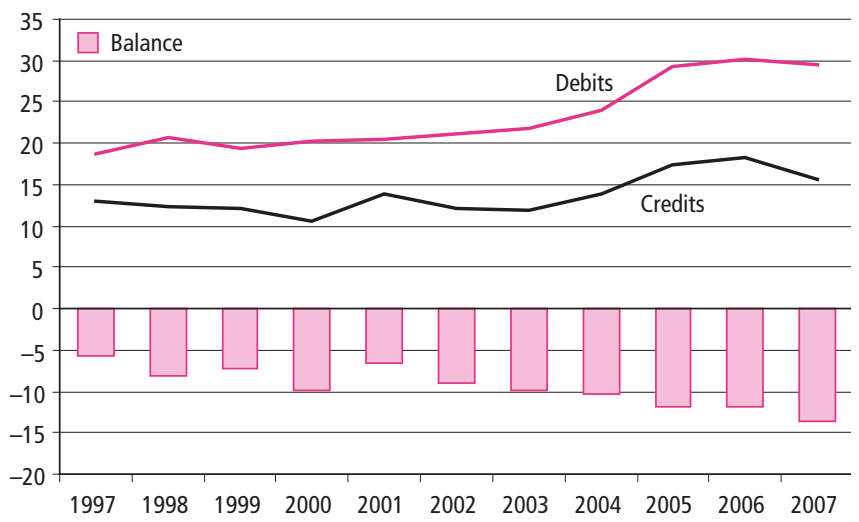

\section{General government transfers}

General government transfers include: taxes and social contributions received from non-resident workers and businesses; current transfers with international organisations (for example, EU Institutions); bilateral aid; social security payments abroad; military grants; and miscellaneous transfers. On the credits side, there was little movement, with the total decreasing by $\mathrm{f0.1}$ billion to $£ 4.2$ billion for 2007. Debits increased slightly by $\mathrm{f0.2}$ billion between 2006 and 2007.

\section{Figure 5.2}

\section{Transfers by general government}

f billion

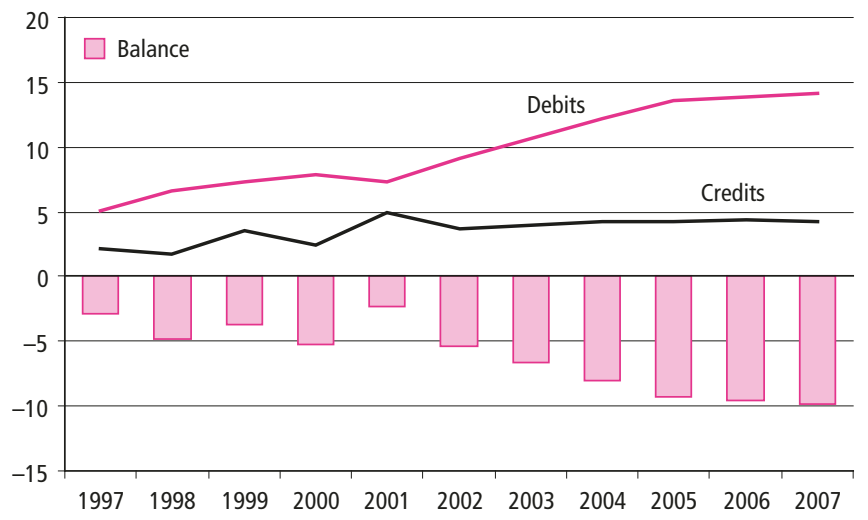

\section{Other sector transfers}

Non-government transfers include those EU transfers where the UK government simply acts as the agent for the final beneficiary (for example, social fund and agricultural guidance fund receipts) or original payer (for example, VAT based contributions). Other sectors transfers also include: taxes on income and wealth paid by UK workers and outward direct investors to foreign governments; insurance premiums and claims; and other transfers (workers remittances, and other private transfers such as gifts). Other sectors credits fell by f2. 5 billion between 2006 and 2007, whilst other sectors debits decreased by $£ 1.0$ billion over the same period. The decreases in both credits and debits in 2007 were both partly due to offsetting falls in net non-life insurance premiums paid to and claims paid by UK companies, following large payments of claims relating to hurricanes in 2005 and 2006. Additionally for credits there were also decreases in receipts from the EU.

\section{Figure 5.3}

\section{Transfers by other sectors}

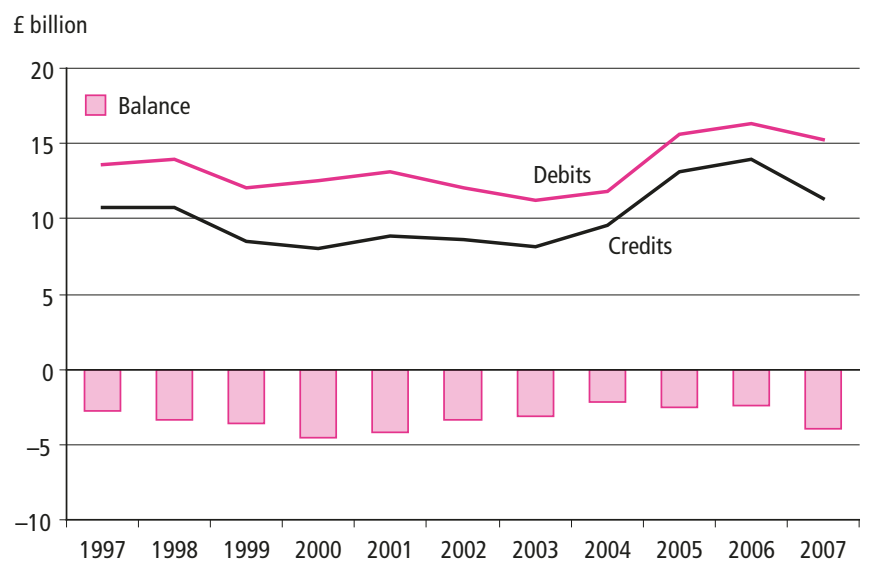




\section{EU institutions}

Transfers with EU institutions constitute the largest single component within current transfers. They showed a deficit in every year from 1986 to 2007; the lowest deficit recorded over the last ten years is $\mathrm{f} 2.3$ billion (in 2001) and the highest deficit $\mathrm{f5.8}$ billion (in 2007). The deficit increased by $\mathrm{f0.9}$ billion between 2006 and 2007.

\section{Figure 5.4}

\section{Transfers with other EU institutions}

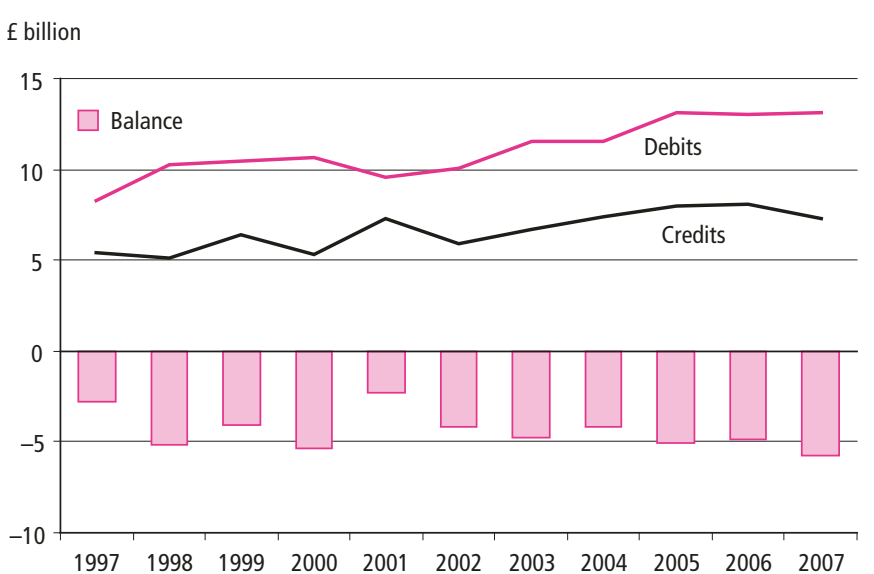




\section{1 cirrent tanster}

$£$ million

\begin{tabular}{|c|c|c|c|c|c|c|c|c|c|c|c|c|}
\hline & & 1997 & 1998 & 1999 & 2000 & 2001 & 2002 & 2003 & 2004 & 2005 & 2006 & 2007 \\
\hline \multicolumn{13}{|l|}{ Credits } \\
\hline \multicolumn{13}{|l|}{ General government } \\
\hline Current taxes on income, wealth etc. & FJKI & 402 & 354 & 337 & 357 & 398 & 527 & 375 & 482 & 546 & 649 & 640 \\
\hline Other taxes on production & FJKH & - & - & - & - & - & - & - & - & - & - & - \\
\hline Other subsidies on production & FJBC & - & - & - & - & - & - & - & - & - & - & - \\
\hline Social contributions & FUBH & 32 & 29 & 29 & 24 & 25 & 24 & 23 & 22 & 22 & 28 & 31 \\
\hline Social benefits & FJBL & - & - & - & - & - & - & - & - & - & - & - \\
\hline \multicolumn{13}{|l|}{ EU Institutions: } \\
\hline (b) Other EU receipts & FKIJ & 6 & 7 & 5 & - & 8 & 13 & 10 & 81 & 71 & 104 & 50 \\
\hline Miscellaneous receipts & FKIK & - & - & - & - & - & - & - & - & - & - & - \\
\hline Total general government & FUUM & 2173 & 1767 & 3542 & 2465 & 4991 & 3663 & 3968 & 4177 & 4294 & 4351 & 4244 \\
\hline \multicolumn{13}{|l|}{ Other sectors } \\
\hline Current taxes on income, wealth etc. & FUBJ & - & - & - & - & - & - & - & - & - & - & - \\
\hline Other taxes on production & FJGC & - & - & - & - & - & - & - & - & - & - & - \\
\hline Other subsidies on production & FJBA & - & - & - & - & - & - & - & - & - & - & - \\
\hline \multicolumn{13}{|l|}{ EU Instititions: } \\
\hline (a) Agricultural Guarantee Fund & EBGL & 3063 & 2935 & 2781 & 2571 & 2336 & 2381 & 2691 & 3315 & 3408 & 3220 & 2943 \\
\hline (b) Social Fund ${ }^{1}$ & H5U2 & 615 & 783 & 434 & 659 & 370 & 412 & 427 & 364 & 842 & 1225 & 749 \\
\hline (c) ECSC Grant & FJKP & 5 & 1 & - & - & 1 & - & - & 2 & _ & - & - \\
\hline Net non-life insurance premiums ${ }^{2}$ & NQQP & 4260 & 4253 & 2495 & 2086 & 3471 & 3008 & 2208 & 3181 & 6133 & 6612 & 5021 \\
\hline Non-life insurance claims ${ }^{3}$ & FJFA & 5 & 7 & 10 & 18 & 25 & 19 & 19 & 47 & 16 & 39 & 50 \\
\hline Other receipts of households ${ }^{4}$ & FKIL & 2712 & 2633 & 2730 & 2653 & 2689 & 2698 & 2713 & 2667 & 2715 & 2748 & 2663 \\
\hline Total other sectors & FUUN & 10735 & 10682 & 8510 & 8018 & 8926 & 8571 & 8079 & 9590 & 13106 & 13872 & 11421 \\
\hline Total & KTND & 12908 & 12449 & 12052 & 10483 & 13917 & 12234 & 12047 & 13767 & 17400 & 18223 & 15665 \\
\hline Of which: Receipts from EU institutions & FKIM & 5422 & 5103 & 6391 & 5314 & 7275 & 5905 & 6688 & 7354 & 7976 & 8119 & 7265 \\
\hline
\end{tabular}

1 Social fund receipts by local government are included up to 2003. From 2004 they are included in general government other EU receipts.

2 Premiums paid to UK insurance companies.

3 Claims paid to UK residents by foreign insurance companies.

4 Includes estimates for workers' remittances and for non-profit institutions serving households. 


\begin{tabular}{|c|c|c|c|c|c|c|c|c|c|c|c|c|}
\hline & & 1997 & 1998 & 1999 & 2000 & 2001 & 2002 & 2003 & 2004 & 2005 & 2006 & 2007 \\
\hline \multicolumn{13}{|l|}{ Debits } \\
\hline \multicolumn{13}{|l|}{ General government } \\
\hline Current taxes on income, wealth etc. & FJKK & - & - & - & - & - & - & - & - & - & - & - \\
\hline Other taxes on production & FJKN & - & - & - & - & - & - & - & - & - & - & - \\
\hline Other subsidies on production & FJCE & - & - & - & - & - & - & - & - & - & - & - \\
\hline Social contributions & FJCH & - & - & - & - & - & - & - & - & - & - & - \\
\hline Social security benefits & FJCK & 1102 & 1162 & 1183 & 1218 & 1292 & 1388 & 1452 & 1596 & 1650 & 1700 & 1829 \\
\hline \multicolumn{13}{|l|}{$\begin{array}{l}\text { Contributions to international organisations } \\
\text { EU Institutions: }\end{array}$} \\
\hline (a) GNP: 4th Resource & HCSO & 2655 & 3516 & 4403 & 4243 & 3859 & 5259 & 6622 & 7565 & 8597 & 8358 & 7996 \\
\hline (b) GNP adjustments & HCSM & -197 & 404 & 229 & 136 & -1 & 76 & 150 & -16 & 135 & 163 & 327 \\
\hline (c) Inter governmental agreements & HCBW & - & - & - & - & - & - & - & - & - & - & - \\
\hline (d) Other & FKIN & 31 & -1 & 11 & 6 & 24 & 10 & 18 & -3 & 106 & 8 & 6 \\
\hline \multicolumn{13}{|l|}{ Other organisations: } \\
\hline (a) Military & HDKF & 168 & 139 & 118 & 157 & 195 & 192 & 152 & 160 & 141 & 159 & 138 \\
\hline (b) Multilateral economic assistance & HCHJ & 268 & 314 & 245 & 503 & 434 & 539 & 367 & 622 & 495 & 945 & 1243 \\
\hline (c) Other & HCKL & 256 & 200 & 268 & 454 & 403 & 248 & 201 & 394 & 550 & 615 & 502 \\
\hline \multicolumn{13}{|l|}{ Bilateral aid: } \\
\hline (a) Non-project grants & FUKT & 131 & 142 & 133 & 175 & 185 & 206 & 268 & 303 & 306 & 300 & 318 \\
\hline Total general government & FUUO & 5087 & 6585 & 7271 & 7778 & 7340 & 9085 & 10657 & 12225 & 13637 & 13853 & 14061 \\
\hline \multicolumn{13}{|l|}{ Other sectors } \\
\hline Current taxes on income, wealth etc. & FJCI & 638 & 454 & 682 & 775 & 523 & 644 & 444 & 535 & 589 & 464 & 633 \\
\hline Other taxes on production & FULB & - & - & - & - & - & - & - & - & - & - & - \\
\hline Other subsidies on production & FJCC & - & - & - & - & - & - & - & - & - & - & - \\
\hline Social contributions & FJBG & - & - & - & - & - & - & - & - & - & - & - \\
\hline Social benefits & FJCM & 75 & 70 & 60 & 31 & 34 & 53 & 21 & 14 & -8 & 28 & -5 \\
\hline \multicolumn{13}{|l|}{ EU Institutions: } \\
\hline (a) Customs duties and agricultural levies & QYRD & 2291 & 2076 & 2024 & 2086 & 2069 & 1919 & 1937 & 2145 & 2237 & 2329 & 2412 \\
\hline (b) Sugar levies & GTBA & 91 & 42 & 46 & 44 & 31 & 25 & 18 & 25 & 24 & - & - \\
\hline (c) VAT based contributions & HCML & 3646 & 3758 & 3920 & 4104 & 3624 & 2720 & 2775 & 1764 & 1980 & 2165 & 2293 \\
\hline (d) VAT adjustments & FSVL & -249 & 470 & -109 & 100 & -49 & 88 & -35 & 25 & 19 & 2 & 26 \\
\hline (e) ECSC Production levy & GTBB & - & - & - & - & - & - & - & - & - & - & - \\
\hline Net non-life insurance premiums ${ }^{1}$ & FJDB & 5 & 7 & 10 & 18 & 25 & 19 & 19 & 47 & 16 & 39 & 50 \\
\hline Non-life insurance claims ${ }^{2}$ & NQQR & 4260 & 4253 & 2495 & 2086 & 3471 & 3008 & 2208 & 3181 & 6133 & 6612 & 5021 \\
\hline Other payments by households ${ }^{3}$ & FKIQ & 2809 & 2906 & 2975 & 3236 & 3364 & 3543 & 3838 & 4082 & 4622 & 4655 & 4868 \\
\hline Total & KTNE & 18653 & 20621 & 19374 & 20258 & 20432 & 21104 & 21882 & 24043 & 29249 & 30147 & 29359 \\
\hline Of which: Payments to EU institutions & FKIR & 8268 & 10265 & 10524 & 10719 & 9557 & 10097 & 11485 & 11505 & 13098 & 13025 & 13060 \\
\hline
\end{tabular}

1 Premiums paid by UK residents to foreign insurance companies.

2 Claims paid by UK insurance companies to non-residents.

3 Includes estimates for workers' remittances and for non-profit institutions serv-

ing households. 


\section{Current transfers}

\begin{tabular}{|c|c|c|c|c|c|c|c|c|c|c|c|c|}
\hline & & 1997 & 1998 & 1999 & 2000 & 2001 & 2002 & 2003 & 2004 & 2005 & 2006 & 2007 \\
\hline \multicolumn{13}{|l|}{ Balances } \\
\hline \multicolumn{13}{|l|}{ General government } \\
\hline Current taxes on income, wealth etc. & FJKJ & 402 & 354 & 337 & 357 & 398 & 527 & 375 & 482 & 546 & 649 & 640 \\
\hline Other taxes on production & FUIZ & - & - & - & - & - & - & - & - & - & - & - \\
\hline Other subsidies on production & FJBD & - & - & - & - & - & - & - & - & - & - & - \\
\hline Social contributions & FUBI & 32 & 29 & 29 & 24 & 25 & 24 & 23 & 22 & 22 & 28 & 31 \\
\hline Social benefits & FJBM & -1102 & -1162 & -1183 & -1218 & -1292 & -1388 & -1452 & -1596 & -1650 & -1700 & -1829 \\
\hline Total general government & FUUQ & -2914 & -4818 & -3729 & -5313 & -2349 & -5422 & -6689 & -8048 & -9343 & -9502 & -9817 \\
\hline \multicolumn{13}{|l|}{ Other sectors } \\
\hline Current taxes on income, wealth etc. & FJHU & -638 & -454 & -682 & -775 & -523 & -644 & -444 & -535 & -589 & -464 & -633 \\
\hline Other taxes on production & FUHT & - & - & - & - & - & - & - & - & - & - & - \\
\hline Other subsidies on production & FUHV & - & - & - & - & - & - & - & - & - & - & - \\
\hline Social contributions & FUHJ & 75 & 70 & 60 & 31 & 34 & 53 & 21 & 14 & -8 & 28 & -5 \\
\hline Social benefits & FJJG & 540 & 713 & 374 & 628 & 336 & 359 & 406 & 350 & 850 & 1197 & 754 \\
\hline Total other sectors & FUUR & -2831 & -3354 & -3593 & -4462 & -4166 & -3448 & -3146 & -2228 & -2506 & -2422 & -3877 \\
\hline Total & KTNF & -5745 & -8172 & -7322 & -9775 & -6515 & -8870 & -9835 & -10276 & -11849 & -11924 & -13694 \\
\hline Of which: EU institutions & FKIS & -2846 & -5162 & -4133 & -5405 & -2282 & -4192 & -4797 & -4151 & -5122 & -4906 & -5795 \\
\hline
\end{tabular}

1 Includes an estimate for workers' remittances. 